\title{
The Design and Preparation of Transparent Hybrid Composite Thin Films with Excellent Optical Properties and Improved Thermal Insulation by Optimized Combination of Nanomaterials
}

\author{
TE HU $\mathbb{1},{ }^{1,2,3,4}$ YUCHANG SU, ${ }^{2}$ IAN R. BAXENDALE,${ }^{3}$ YAPING ZHANG, ${ }^{1}$ \\ and JINFAN ZHU ${ }^{2}$ \\ 1.-Faculty of Science, Kunming University of Science and Technology, South Jingming Road, \\ Kunming 650500, China. 2.-School of Materials Science and Engineering, Central South \\ University, South Lushan Road, Changsha 410083, China. 3.-Department of Chemistry, Durham \\ University, South Road, Durham DH1 3LE, UK. 4.—e-mail: hutete@csu.edu.cn
}

For a single nano-optical material, it is difficult to possess high transmittance and adequately filter ultraviolet (UV) and infrared radiation (IR) simultaneously. Consequently, hybrid nano-optical materials comprising components of appropriate proportions for superimposing serviceable optical property are required. The design, optimization and processing of new composite blends with an aim to creating defect free thin films is far from a trivial endeavor. In this report, optimum composition and optical properties of hybrid nano-optical material has been determined and improved by crossover matching experiments and ball milling, respectively. Film preparation has been optimized to reduce defects expressed as cracks, tiny bubbles, strips, groove points, corrugation, and formation of acicular fibers by regulating proportion of polyvinyl butyral colloid and dry film processes. Two ameliorative processing conditions are exemplified where the resultant composite films possessed $86 \%$ maximum transmittance in the visible range and $90 \%$ and $50 \%$ blocking rate with respect to the IR and UV bands.

Key words: Nano-optical materials, transparent thermal insulation, composite functional thin film, optical property, ball milling, colloid, film defect

\section{INTRODUCTION}

Energy efficacy in the modern world is of paramount importance. Human artificial environmental climate control of buildings and transportation systems through the utilization of air conditioning (AC) systems consumes considerable levels of the world's energy resources and is thus indicated as a significant contributor of greenhouse gas emissions. ${ }^{1}$ Solutions devised to help reduce energy consumption such as the introduction of intelligent AC control systems with evolutionary algorithms ${ }^{2}$ and the construction of new buildings and vehicles

(Received June 26, 2019; accepted December 7, 2019; published online December 18, 2019) incorporating renewable and green energy sources such as photovoltaic (PV), ${ }^{3}$ fuel cells (FC) ${ }^{4}$ and reservoir $\mathrm{Li}$-ion batteries ${ }^{5}$ is being trialed. However, the current low conversion efficiencies and often inadequate on-demand peak power output from these new energy sources ${ }^{6,7}$ means such additions do not constitute a complete and practical solution. Furthermore, the complexities of monitoring and instantaneously responding to continuous changes in the building occupancy as well as more demanding external environmental factors such as daily weather patterns or the rapidly fluctuating levels of incident solar radiation are especially challenging. ${ }^{6-8}$ Indeed, due to their aesthetic appeal, most buildings and vehicles (including the need for effective vision) are designed with a natural abundance of windows which can magnify the issue of 
interior climate control due to direct transmission of solar radiation. To ameliorate much of the negative impact, composite functional thin films can be used to coat the glass surfaces acting to block incident 'heating' ultraviolet (UV) and infrared radiation (IR) bands but still allow visible light through. These wavelength selective coatings thereby function as thermal insulation importantly helping to control interior temperatures and thereby reduce energy consumption from AC usage.

This is a very active area of research with obvious high commercial impact and, although a great deal of progress has been made, there is still room for improvement, especially with respect to cost reduction of the nano-materials used. ${ }^{9}$ One important consideration from the numerous previous studies is that it is extremely difficult to find a single component material that possesses the necessary optical properties (coverage of the desired exclusion UV and IR bands) to subsequently make viable composite films. A simple solution to this issue is to investigate hybrid nano-optical materials which together provide a combined optical profile matching the desired optical blocking window for the solar light.

In this paper, we selected cesium-doped tungsten oxide (CTO $)^{9,10}$ and lanthanum boride $\left(\mathrm{LaB}_{6}\right)^{11,12}$ to prepare hybrid materials with extrapolated optical properties through orthogonal translation experiments from graphene. ${ }^{13,14}$ Different films comprised of cesium-doped tungsten oxide (CTO), lanthanum boride $\left(\mathrm{LaB}_{6}\right)$, antimony-doped tin oxide (ATO), ${ }^{15,16}$ tin-doped indium oxide (ITO $)^{17,18}$ and antimony and tin-doped indium oxide (IATO) ${ }^{19,20}$ were synthesized by adjusting the proportion of polyvinyl butyral (PVB), ${ }^{21,22}$ dibutyl phthalate $(\mathrm{DBP})^{23}$ and solvents (ethanol, ethyl acetate and acetylacetone) whilst optimizing the film forming conditions. The resulting films were characterized and analyzed for their optical properties in which the best one has obtained $86 \%$ maximum transmittance in visible light range, $90 \%$ and $50 \%$ blocking rate on UV and IR and SHGC as 0.61 .

\section{MATERIALS AND METHODS}

\section{Experimental Overview}

Combination of $\mathrm{LaB}_{6}$ and CTO with mass ratio 3:2 possesses optimal optical property effectively blocking UV and IR ranges and high transmittance in the visible light zone, of which the component ratio was decided by crossover matching experiments according to optical features of six nano-optical materials and optical characteristics was optimized by a ball grinding miller improving size and morphology of nanoparticles. Each nano-optical material was diffused in ethanol by using an ultrasonicator to treat for $30 \mathrm{~min}$ and employing a magnetic stirrer to stir for $1 \mathrm{~h}$, then optical characteristics of the corresponding dispersion solution was characterized by the use of a spectrophotometer. In addition, phase composition and microstructure of each nano-optical material were observed by $\mathrm{x}$-ray diffraction patterns (XRD) and a transmission electron microscope (TEM) severally.

Components of PVB colloid or composite colloid for preparing a thin film without defects were determined by searching out optimum value from variation of a viscosity or an optical property by contrastive experiments. PVB colloid was obtained through 10 wt.\% PVB and 10 wt.\% DBP were completely dissolved in mixed solution with ethanol and acetylacetone in mass ratio $3: 5$ by magnetic stirring for $6 \mathrm{~h}$ at ambient, after that, composite colloid was obtained when 3 wt.\% hybrid nanooptical material was homogenously diffused in the PVB colloid by magnetic stirring for $24 \mathrm{~h}$, ultrasonic oscillating for $2 \mathrm{~h}$ and standing for $3 \mathrm{~h}$. A piece of composite film (size as $47 \mathrm{~mm} \times 23 \mathrm{~mm}$ and thickness as $0.2 \mathrm{~mm}$ ) was formed in terms of following steps: a layer of the composite colloid (size as $47 \mathrm{~mm} \times 23 \mathrm{~mm}$ and thickness as $1 \mathrm{~mm}$ ) was evenly applied on a plexiglass using an adjustable film applicator; the sample was dried in air for 60-120 min at ambient temperature; subsequently, it was placed in a vacuum oven at $40-60^{\circ} \mathrm{C}$ no vacuum for drying 15-20 min; and then turning the vacuum on $0 \mathrm{MPa}$ no heating working for $15-$ 30 min when the edge of the coating had been began to shrink.

Ultimately, surface morphology and the optical property of the synthesized composite film were characterized by a scanning electron microscope (SEM) and a solar film tester.

\section{Characterization Analysis of the Nano-optical Materials}

Figure 1 shows the $x$-ray diffraction patterns (XRD) of six nano-optical materials demonstrating their pure phases without impurities. Transmission electron microscope (TEM) images of the materials are presented in Fig. 2. Figure 3 illustrates the

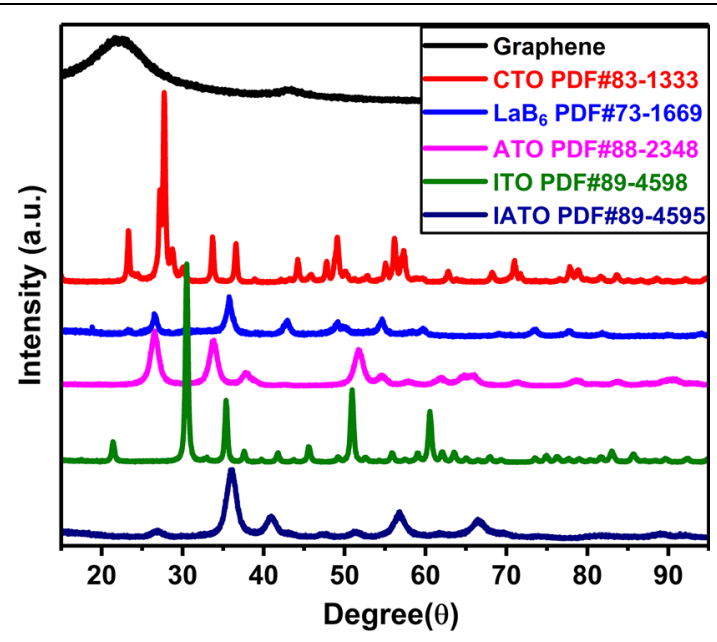

Fig. 1. XRD patterns of the investigated optical materials. 

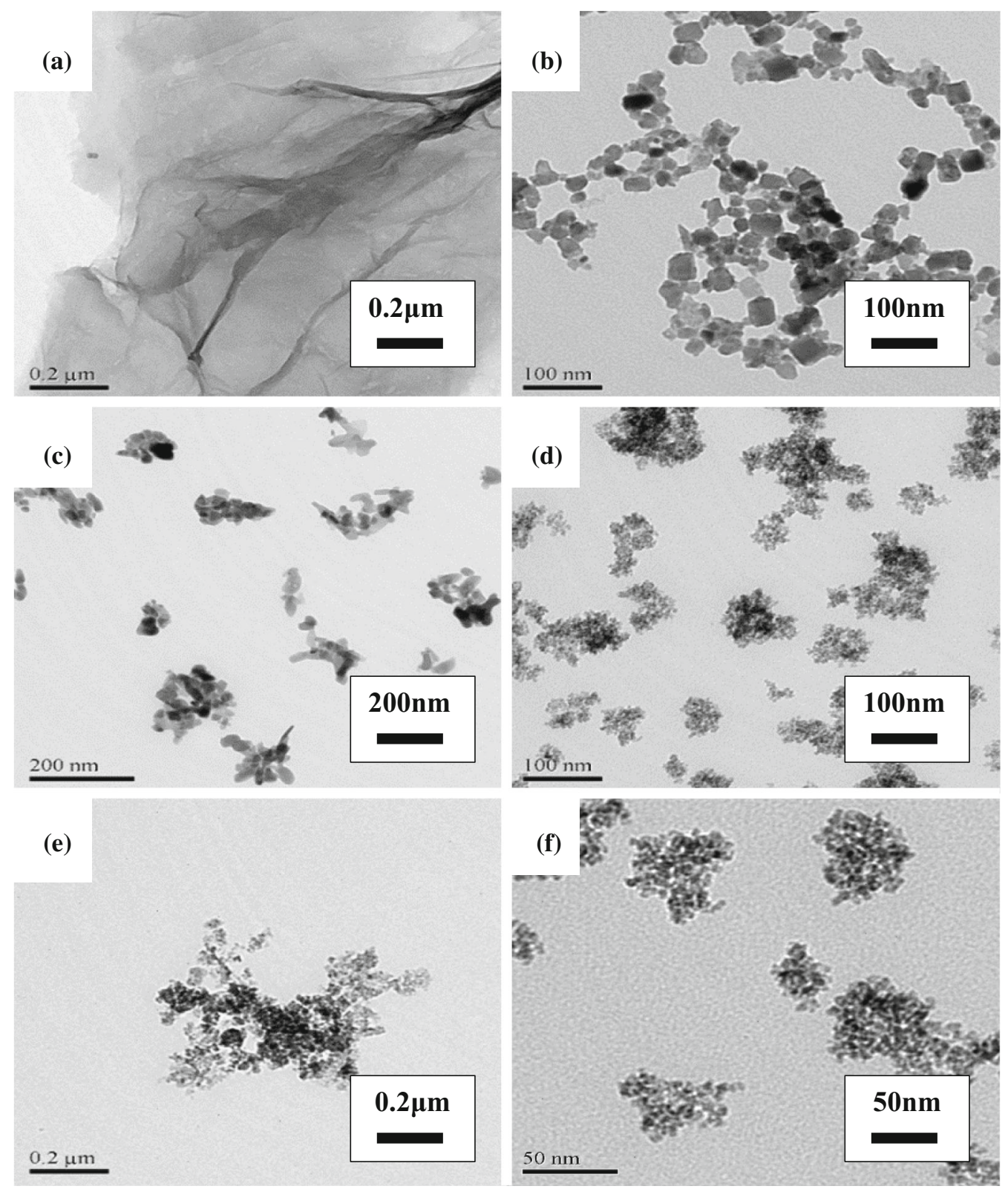

(d)
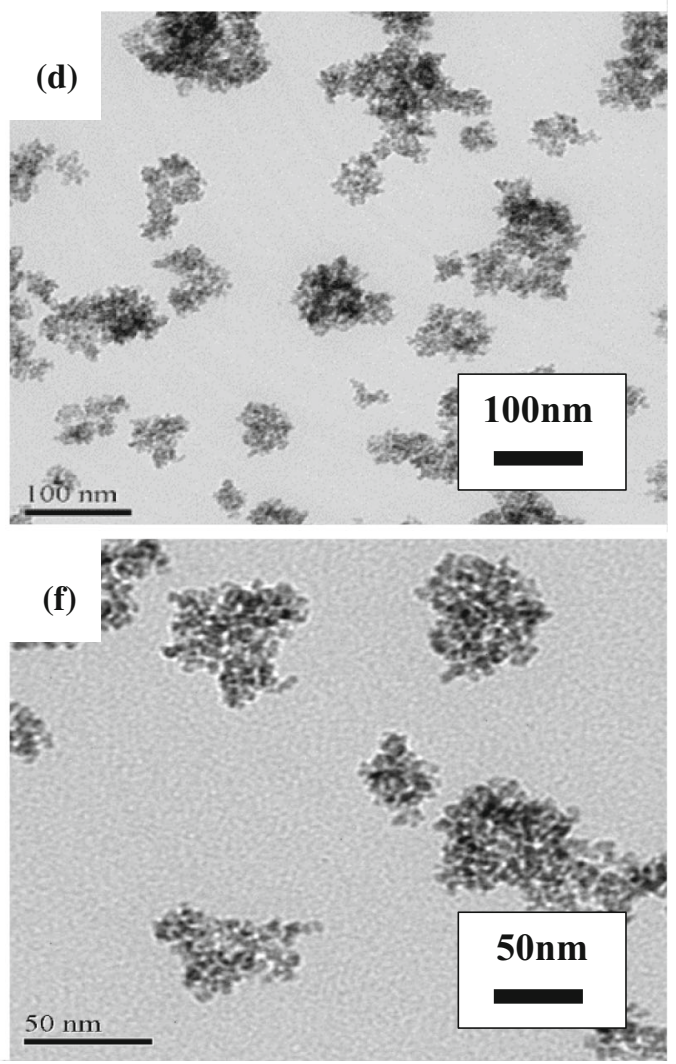

Fig. 2. TEM images of the six nano-optical materials: (a) Graphene, (b) CTO, (c) LaB 6 , (d) ATO, (e) ITO, (f) IATO.

relationship of the optical properties and the solid dispersions of the materials in ethanol.

In Fig. 3a, the transmittance of graphene steadily rises in an arc covering the range of 375-1020 nm evaluated. According to Fig. $3 \mathrm{~b}$, the transmissivity of CTO increases from $\sim 650 \mathrm{~nm}$, with a peak on $830 \mathrm{~nm}$, then dropping down steadily to $1200 \mathrm{~nm}$. The optical property of $\mathrm{LaB}_{6}$ is shown in Fig. 3c, the transmittance is more translocated to a wavelength maximum of $\sim 600 \mathrm{~nm}$ and has a much flatter appearance. ATO and ITO have similar optical properties as presented in Fig. $3 \mathrm{~d}$ and e, their transmittance increases from $380 \mathrm{~nm}$ to $1200 \mathrm{~nm}$ to give an apex at the end of the range. Based on Fig. 3f, the transmittance of IATO increases from
$380 \mathrm{~nm}$ to a maximum of $\sim 915 \mathrm{~nm}$, thereafter decreasing again to the recorded range of $1020 \mathrm{~nm}$.

\section{RESULT AND DISCUSSION}

\section{Composition of Hybrid Materials}

The desired optical properties of the materials are for maximum transmittance located in the visible region and effective blocking covering the UV and broader IR regions. As Fig. 3a clearly shows, graphene is not a good choice with regards to its ability to selectively filter the undesirable wavelengths. The compounds $\mathrm{LaB}_{6}$ and CTO partially block the UV and IR zones and their transmittance apexes are in, or are closer to, the visible light range, therefore, they are more suitable candidates. 

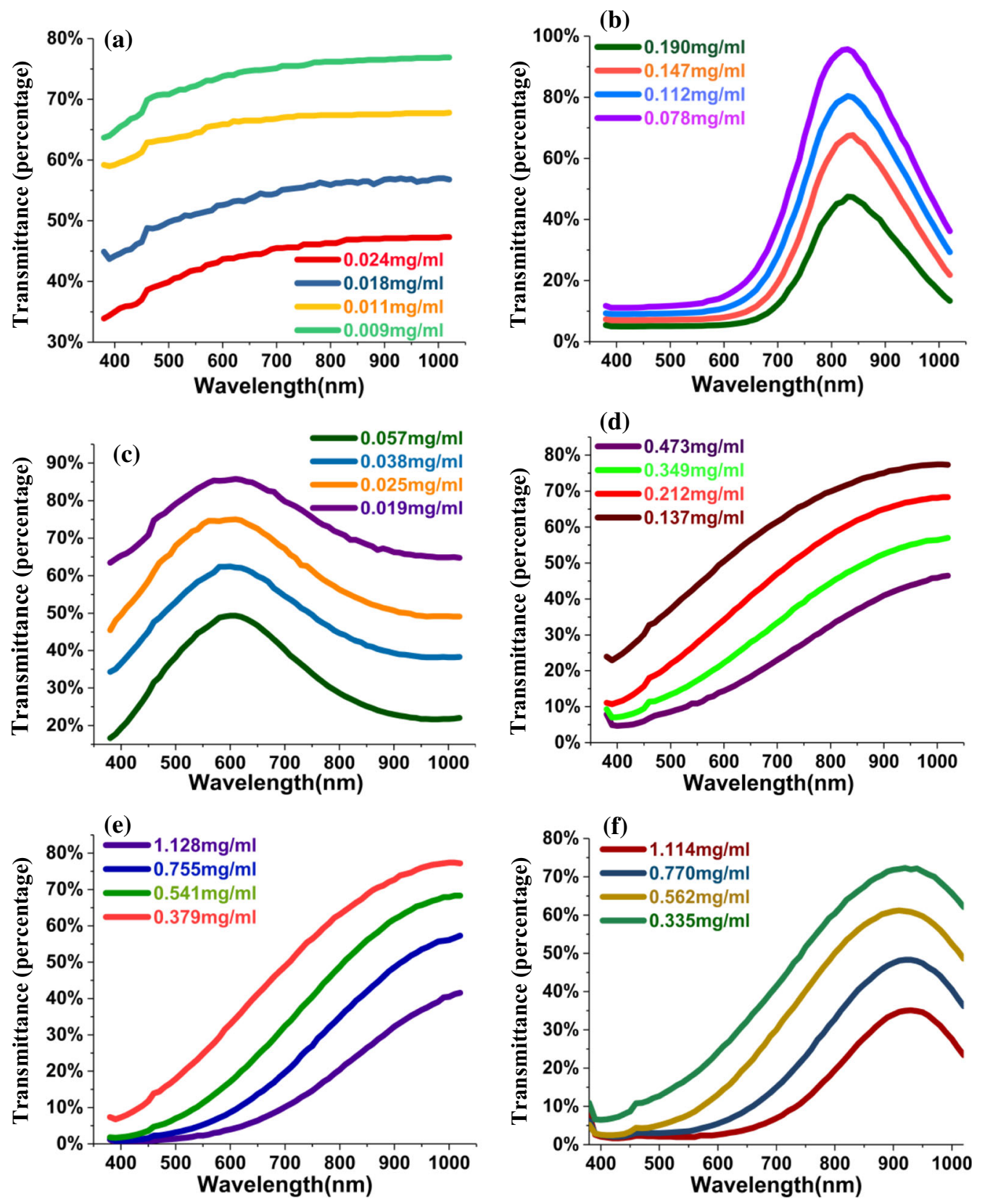

Fig. 3. Transmittance of original six nano-optical materials with various concentrations: (a) Graphene, (b) CTO, (c) LaB ${ }_{6}$, (d) ATO, (e) ITO, (f) IATO.

However, their specific blocking and transmittance characteristics are not ideal and so they were further evaluated in combination with the other four evaluated materials in dual or multiple crossover experiments (varying weight proportions).

The dual matching experimental results are shown in Fig. 4. The maximum transmittance of the hybrid nano-optical materials in general was widened (Fig. 4a1 and b1). Also, when $\mathrm{LaB}_{6}$ and CTO were separately matched with ATO, ITO and IATO they created a general shift to the left side on $\mathrm{LaB}_{6}$ or right side on CTO as seen in Fig. $4 \mathrm{a} 2-\mathrm{b} 4$. From these results a further investigation was undertaken to optimize the optical window by doping of $\mathrm{LaB}_{6}$ and $\mathrm{CTO}$ in a triple combination with ATO, ITO and IATO. Based upon the optical properties of the original combinations in Fig. 4a1 and b1, optimal mass ratio of $\mathrm{LaB}_{6}$ and CTO was calculated as 3:2. Additionally, before generating the ternary composites, all materials underwent ball milling to improve their physical forms for blending.

A planetary ball miller was used to blend at different weight ratios of the nano-powder mixtures to zirconia balls and ethylenediaminetetraacetic acid (EDTA). ${ }^{24}$ Relative ratios of $1: 50$ and 1:0.3, 
(a1)
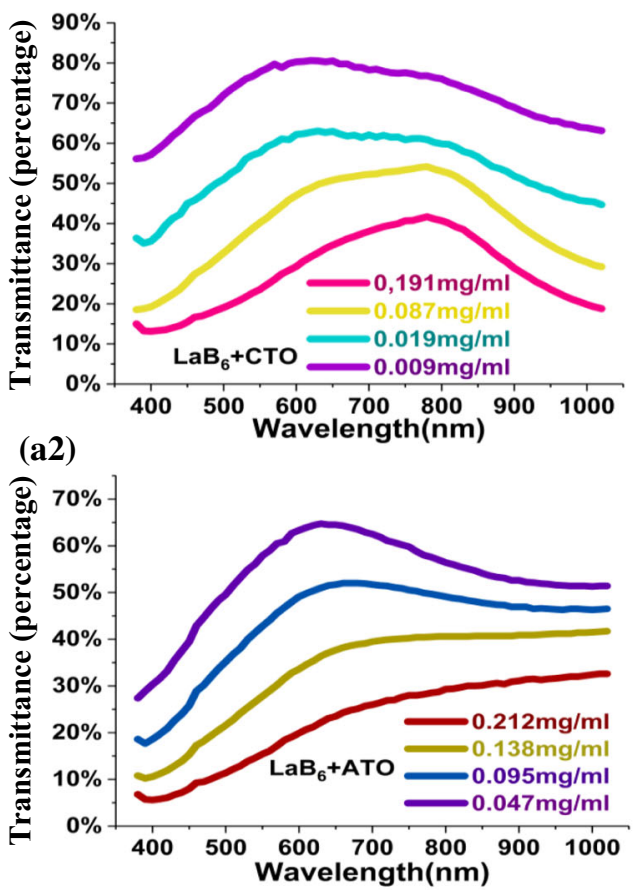

(a3)
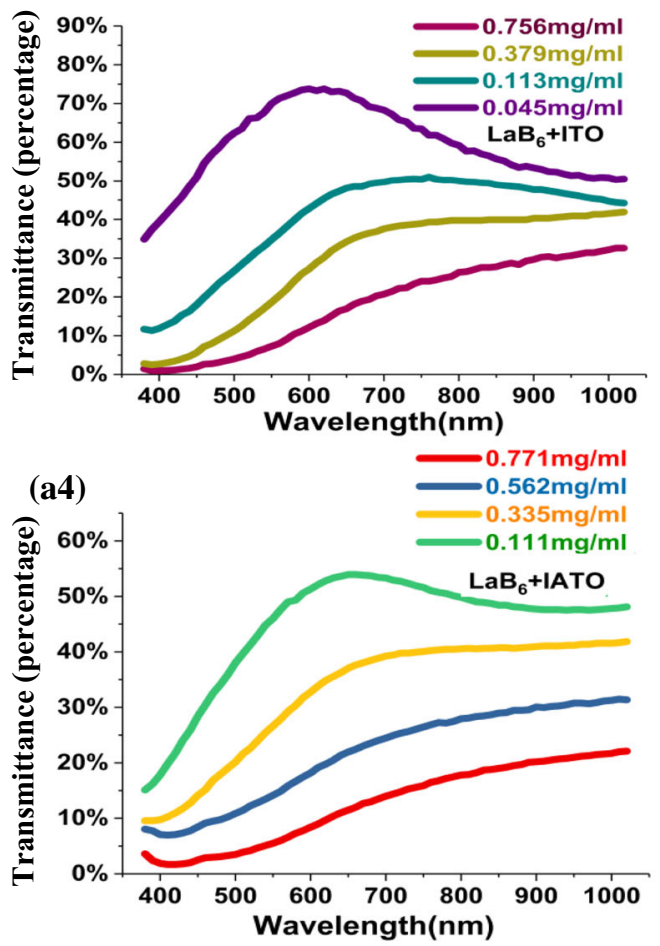
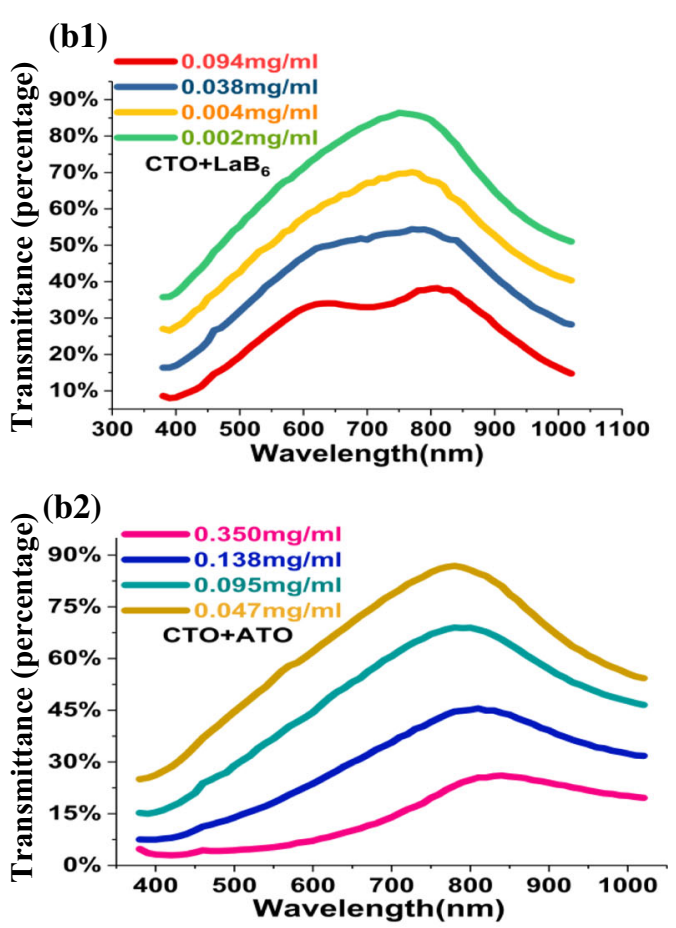

(b3)

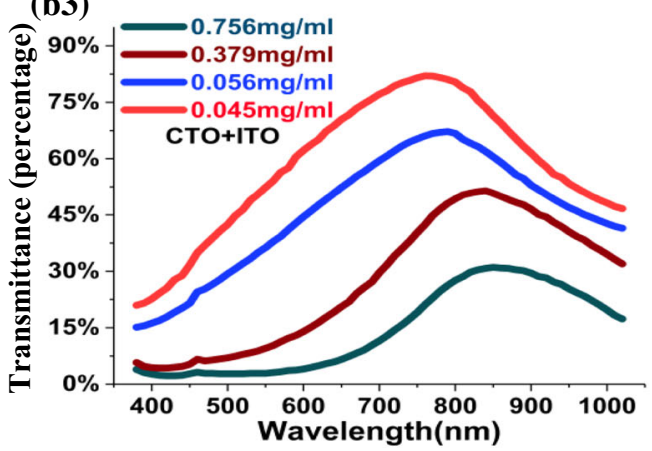

(b4)

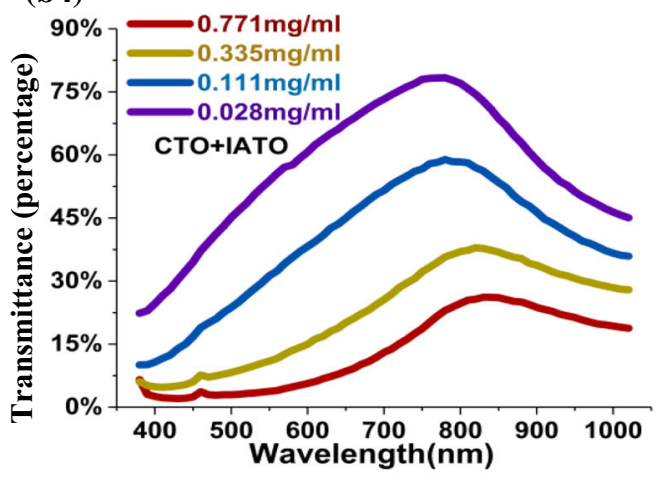

Fig. 4. Transmittance of mixtures when $\mathrm{LaB}_{6}$ and $\mathrm{CTO}$ as basic material to match with other four nano-optical materials under diverse concentrations: (a1), (a2), (a3) and (a4) $\mathrm{LaB}_{6}$ mixed with other four nano-optical materials, (b1), (b2), (b3) and (b4) CTO mixed with other four nano-optical materials. 


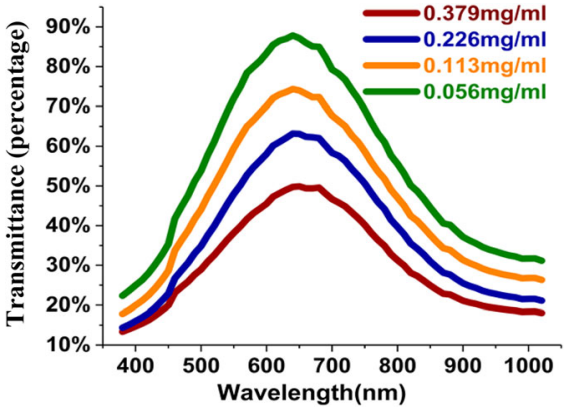

Fig. 5. Transmittance of mixture of $\mathrm{LaB}_{6}$ and $\mathrm{CTO}$ with mass ratio 3:2 after ball milling under different concentrations. were used and ethanol added to generate specific concentrations in the aluminum oxide jar $(250 \mathrm{~mL})$, the contents were then subjected to high energy collision. A combination of milling balls was used varying in sizes $10 \mathrm{~mm}$ diameter (large), $8 \mathrm{~mm}$ diameter (medium) and $5 \mathrm{~mm}$ diameter (small) and these were applied in mass proportion 3:2:1. During processing, the jars were rotated for up to $72 \mathrm{~h}$ at the constant milling speed of $300 \mathrm{rpm}$ and the rotational direction was changed every $15 \mathrm{~min}$.

Variations in the lattice strain, distortion and particulate spacing in nanomaterials were expected due to the ball milling. ${ }^{25}$ However, unexpectedly the
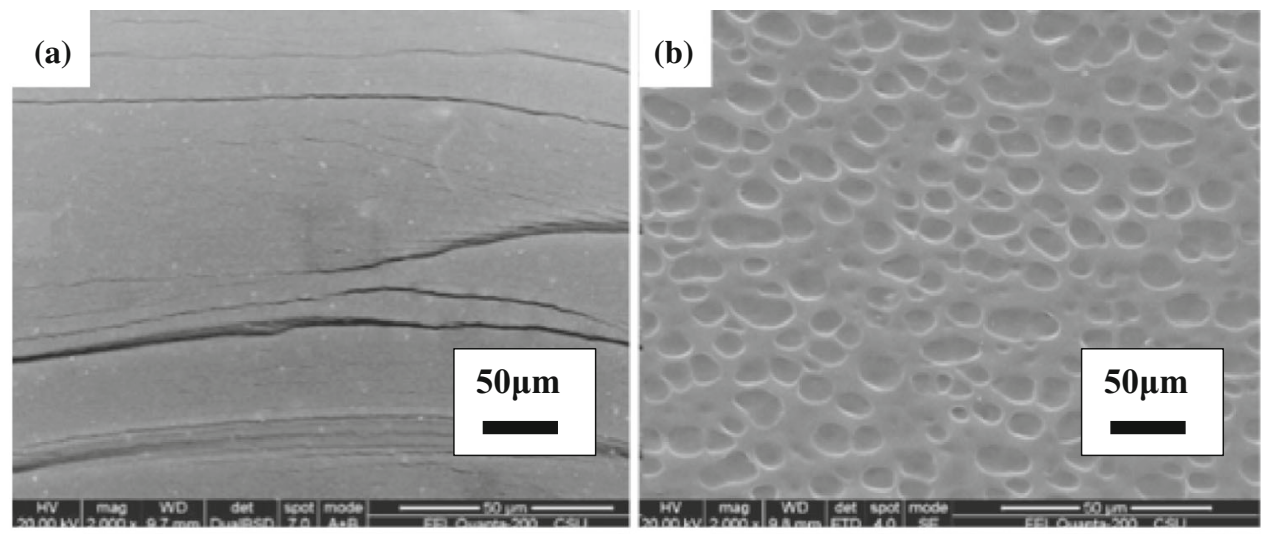

(c)
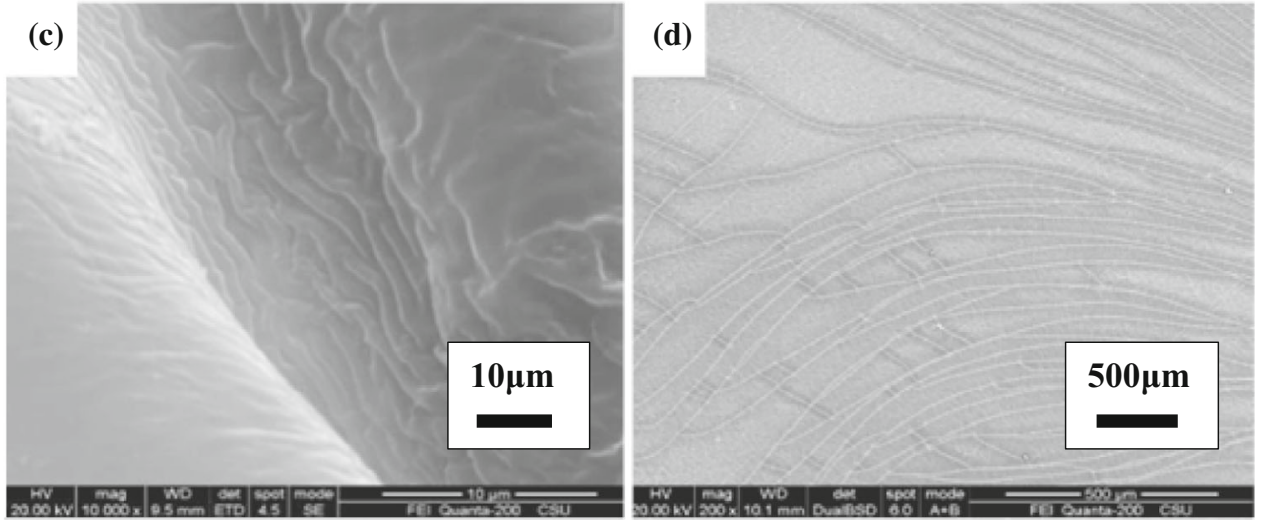

(e)

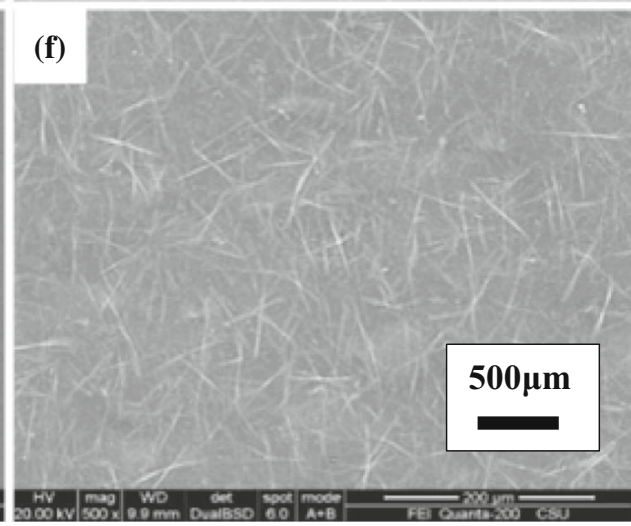

Fig. 6. Film defects from different preparation conditions: (a) Cracks of film surface, (b) Groove points of film surface, (c) corrugation of film bottom, (d) stripes of film surface and bottom, (e) bubbles of film surface, (f) acicular fiber. 
optical property of the milled materials exhibited analogous Gaussian distribution as shown in Fig. 5. The maximum transmittance was centered around $640 \mathrm{~nm}$ in the middle of visible light range with rapid tail off towards the UV and IR regions providing approximately $80 \%$ and $70 \%$ cut offs, hence providing a suitable hybrid material to synthesize composite thin film.

\section{Controlled Formation of PVB Thin Film}

It is well known that even minor changes to the components of the PVB colloid and inappropriate film drying procedures will result in various film defects. $^{26-28}$

These film defects make the film give rise to haziness, abnormal scattering, hardly adhering on substrate, easily appearing as various deformations, poor appearance and so on that will result in extremely deceased optical properties of composite film for thermal insulation and transmittance. Therefore, for removing them, then formation reasons for these film defects have to be revealed to obtain optimal optical properties of the composite film.

During the preparation of the PVB thin films, six classes of defects were revealed as shown in Fig. 6. Specifically, cracks in the film surface were recorded (Fig. 6a) which was formed under laboratory ambient or vacuum oven drying over longer time periods $(>2 \mathrm{~h})$ or higher drying temperatures $\left(>60^{\circ} \mathrm{C}\right)$. We also noted that when atmospheric moisture deposited onto the film surface, groove points were produced as shown in Fig. 6b. Restricted volatilization of the organic solvents on the film bottom resulted in corrugations in the film as depicted in Fig. 6c. In general, although film forming speed was accelerated when higher PVB content was used in the deposition colloid this also led to the formation of strips in the film surface; Fig. 6d. Also care had to be taken in solubilizing the PVB in the solvent mixture as the action of the magnetic stirrer created microbubbles, which in the film gradually moved to the surface and merged with each other to create larger bubbles on the film surface; Fig. 6e. To control solvent volatilization speed, DBP (dibutyl phthalate) was added as a plasticizer to the PVB colloid but adding much higher content gave rise to acicular fiber in the sample; Fig. $6 f$.

The most important parameter determined from contrast experiments to achieving a defect free film was the rate of solvent volatilization; this was controlled for by adjusting the viscosity of PVB colloid and exacting drying conditions. The specific details of the protocol will be discussed below.

Figure 7 illustrates that the viscosity of the PVB colloid was increased when the proportion of the PVB in the solvent mixture was increased which

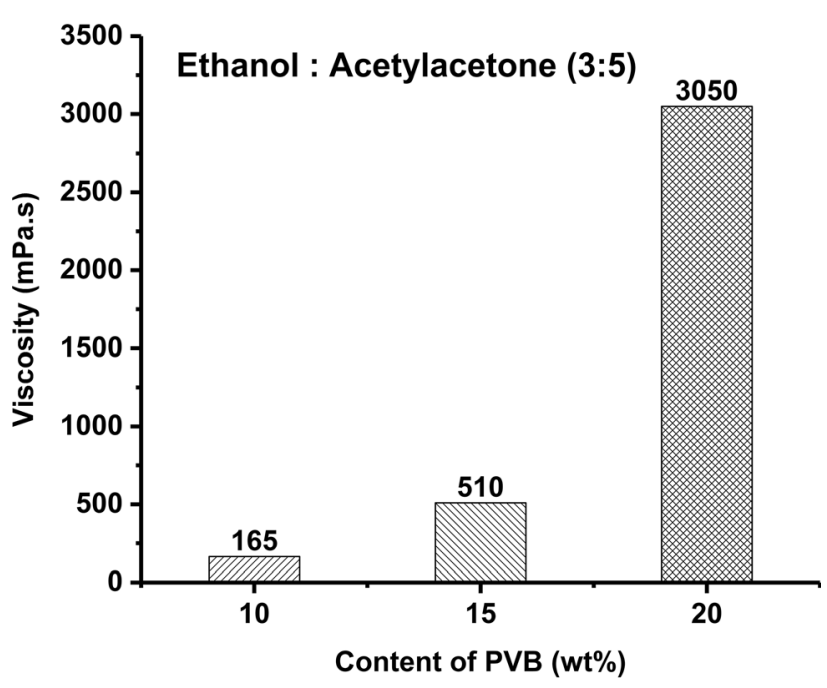

Fig. 7. Viscosity of the PVB colloid with different content of PVB.

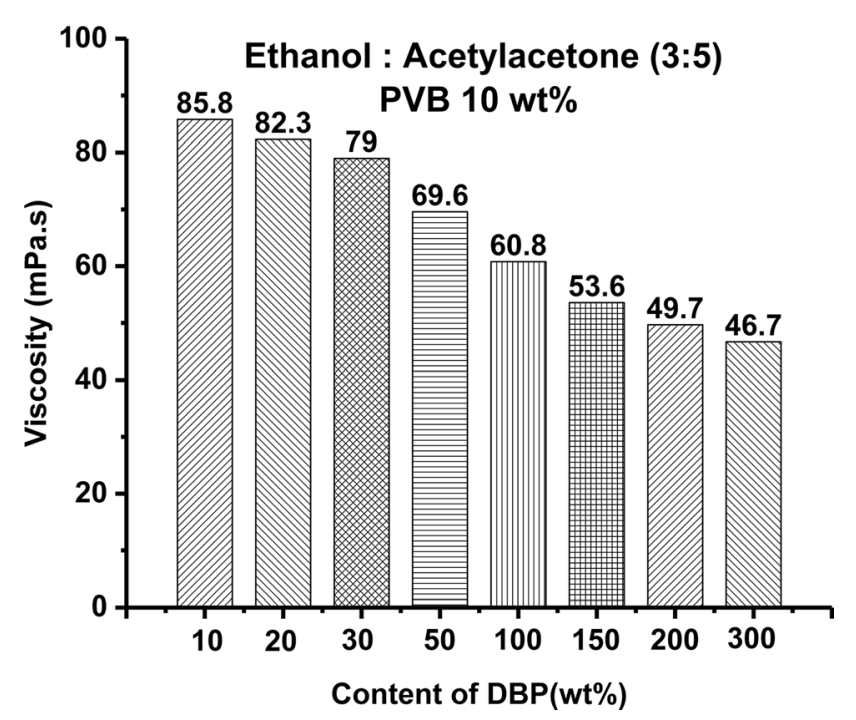

Fig. 8. Viscosity of PVB colloid when different contents of DBP.

resulted in formation of cracks, tiny bubbles and strips. Therefore, a lower content of $10 \mathrm{wt} \%$ PVB was employed. Corrugation on the PVB film bottom was adjusted by the addition of the plasticizer DBP in Fig. 8. However, the coated colloid on plexiglass gave issues in forming a quality film, groove points emerged on film surface and acicular fibers were also seen and attributed to the decreased solubility of the PVB in mixture solvent when content of the DBP was high, consequently lower content of 10 wt.\% DBP was also employed.

In addition to optimizing the component proportion of PVB colloid, elimination of defects from the PVB films required careful regulation of the drying procedures to control the solvent volatilization, especially between the film surface and the lower 


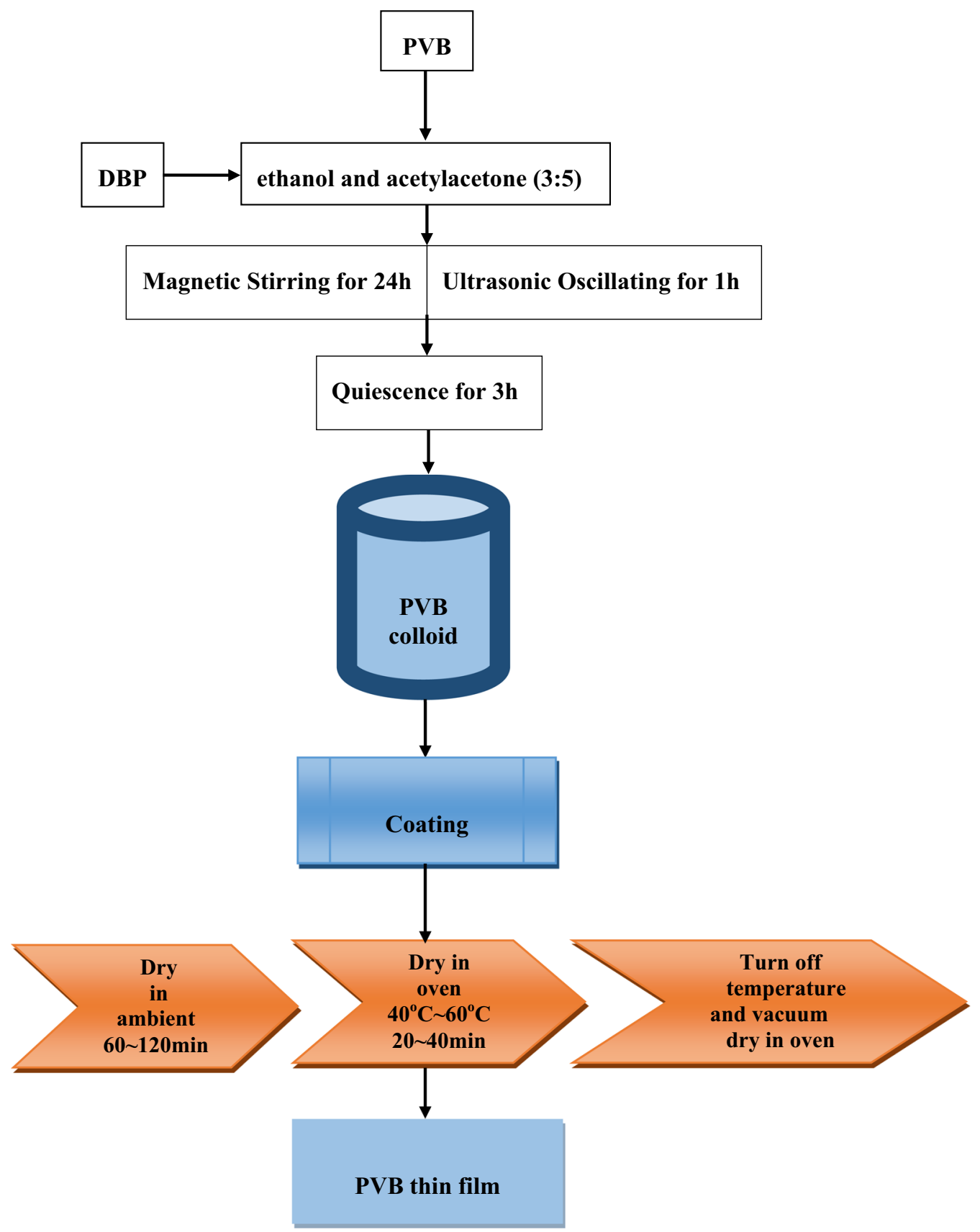

Fig. 9. Preparation process of PVB thin film without film defects.

bonding surface, a general schematic of the entire process is shown in Fig. 9 and a typical SEM image of the resulting smooth and uniform PVB film without defects is exhibited in Fig. 10.

\section{Preparation of Composite Functional Thin Film}

Refer to Fig. 10, as composite thin films were prepared by blending various solid content hybrid nano-optical materials with PVB colloid under the specified drying conditions. The assembly process is divided into two major steps; (1) constitution of composite colloid and (2) formation of composite film.

According to contrast experiments, the viscosity of the PVB colloid composite was higher without added DBP. The specific preparation process involved mixing ethanol and acetylacetone in mass ratio $3: 5$ in a $100 \mathrm{ml}$ beaker which was covered by plastic wrap and stirred via a magnetic stirrer. To the mixture was added in batches $10 \mathrm{wt} \%$ PVB and 10 wt.\% DBP until the mixture completely dissolved. The bulk material was then equably separated into four portions and respectively dispersed with the hybrid nano-optical materials in $3 \mathrm{wt} . \%$, 


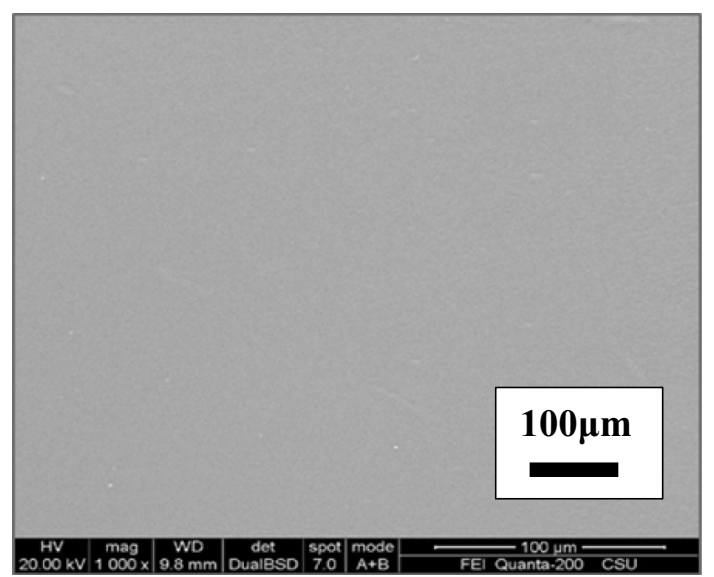

Fig. 10. SEM image of PVB thin film without film defects.

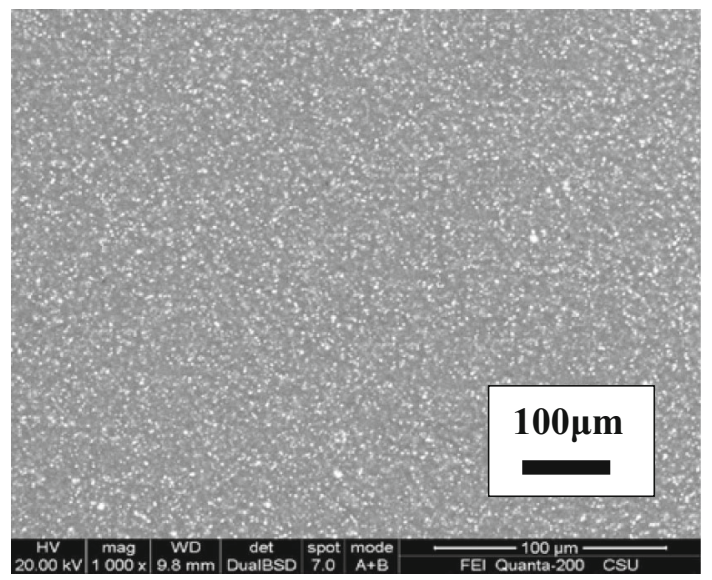

Fig. 11. SEM image of composite thin film without film defects.

4 wt. $\%, 5$ wt. $\%$ and 6 wt.\% solid contents. Finally, the mixtures were ultrasonicated for $1 \mathrm{~h}$ and then in quiescence for $24 \mathrm{~h}$ until all the microbubbles had evolved from composite colloid.

The preparation of the films was conducted by laying down a thin line of the composite colloid and then evenly smearing across the surface of a sheet of plexiglass (Size is $47 \mathrm{~mm} \times 23 \mathrm{~mm}$ and thickness is $1 \mathrm{~mm}$ ) through the use of an applicator. The samples were then air dried for 60-120 min at ambient temperature, until the edge of the coating had shrunk then they were placed in a vacuum oven at $40-60^{\circ} \mathrm{C}$ for $15-20 \mathrm{~min}$, and then the vacuum applied (0 MPa) without heating for 15-30 min.

Hybrid nano-optical materials were homogenously diffused in the film that had been observed from SEM images of composite film in Fig. 11. Four composite thin films with 6 wt.\%, 5 wt. $\%, 4$ wt. $\%$ and $3 \mathrm{wt} . \%$ solid contents were used as cover sheets for the blue logo as shown in Fig. 12a, b, c, and d.

Vital optical parameters of these films on specific wavelengths were characterized by a solar film tester as in Fig. 13. Solid content of hybrid nanooptical materials in the composite films was found to be in direct proportion to the thermal isolation effectiveness that can be expressed by thevsolar heat gain coefficient (SHGC) and in inverse proportion to transmittance of visible light. Consequently, it is hard to achieve a film that possesses higher transmittance in the visible light range and stronger rejection rates in the UV and IR concurrently. However, comparing the three composite films, the 4th film with 3 wt.\% solid content obtains a high transmittance in the visible light range of $86 \%$, and inhibition of UV and IR at $90 \%$ and $50 \%$, respectively. Its SHGC is 0.61 providing a coating that satisfies the application requirements of transparent thermal insulation on a window of a building and a vehicle.

\section{CONCLUSION}

New composite functional thin films with transparent thermal insulation for use in energy conservation and emission reduction are continuously and intensively attracting the attention of researchers around the world. Single component nano-optical materials cannot directly meet the expected optical characteristics of such film, whereas, a quite effective method is to design hybrid nano-optical materials to superimpose individual serviceable optical characteristics to achieve the desired photo-optical properties.

In this paper, $\mathrm{LaB}_{6}$ and $\mathrm{CTO}$ with a mass ratio of $3: 2$ as optimal composition of hybrid nano-optical materials was designed and by crossover screening experiments of graphene, $\mathrm{CTO}, \mathrm{LaB}_{6}, \mathrm{ATO}, \mathrm{ITO}$ and IATO and lattice strain, distortion and spacing in nanoparticles were changed by ball milling to obtain a decent optical property of $90 \%$ transmittance on $640 \mathrm{~nm}$ in a visible wavelength and $80 \%$ and $70 \%$ blocking rate on UV and IR. In addition, pure PVB film without defects such as cracks, tiny bubbles, strips, groove points, corrugation and acicular fiber was prepared by adjusting the content ratio of diverse compositions in PVB colloid and dry film procedures. Under these two optimized conditions, composite functional thin film with 3 wt. $\%$ solid content possesses $86 \%$ maximum transmittance in the visible light range, $90 \%$ and $50 \%$ blocking rate on UV and IR and SHGC as 0.61 that achieved transparent thermal insulation and can be applied on windows of a building and vehicles. 

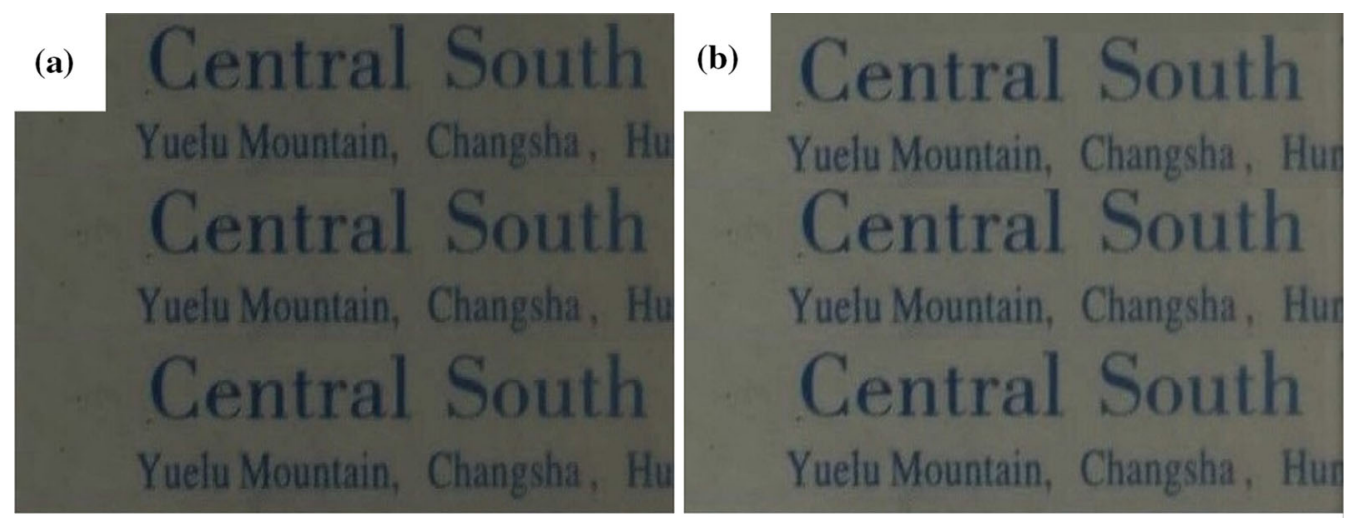

\section{(c)}

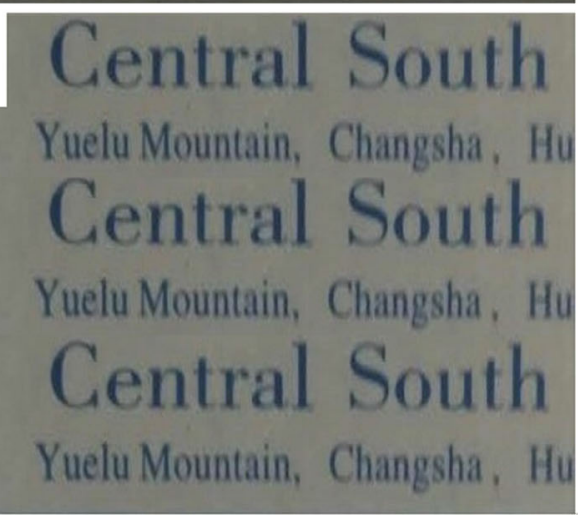

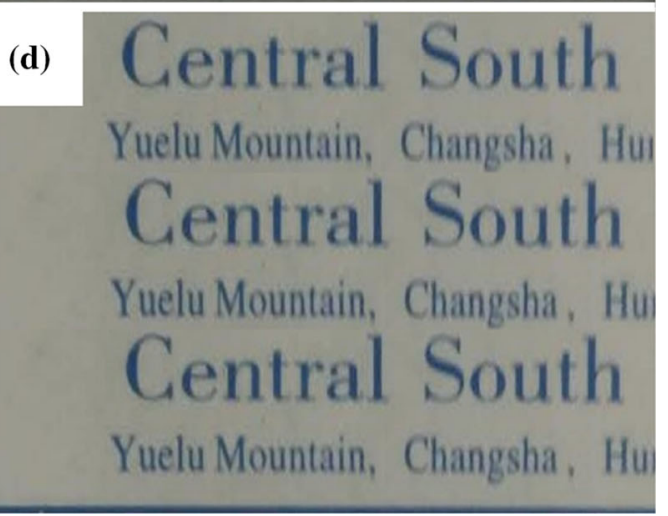

Fig. 12. Images of composite thin films with different proportion hybrid nano-optical materials: (a) 6 wt.\% (1\# film), (b) 5 wt.\% (2\# film), (c) 4 wt.\% (3\# film) and (d) 3 wt.\% (4\# film).

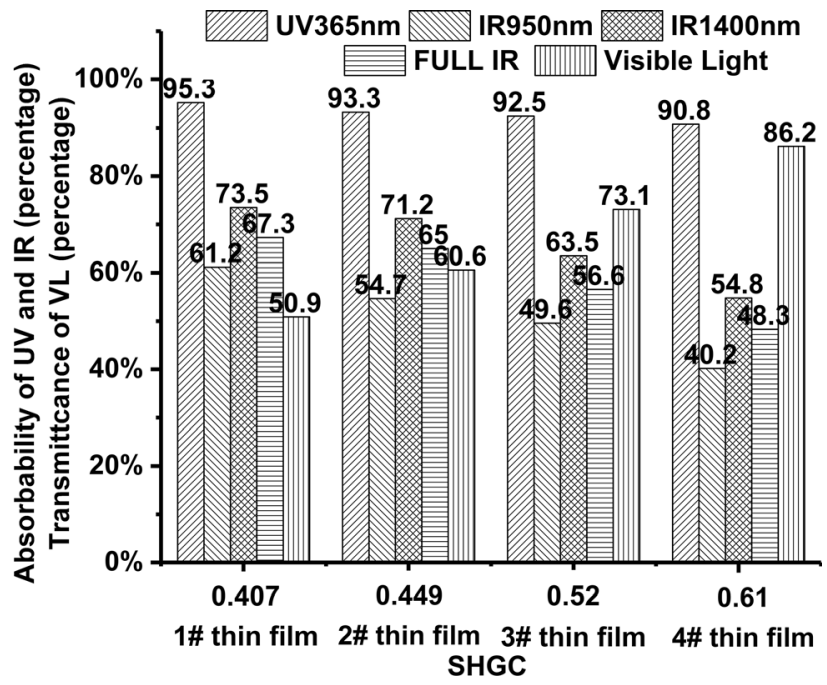

Fig. 13. Optical properties of composite thin films with diverse content hybrid nano materials (3 wt.\%, 4 wt. $\%, 5$ wt. $\%$ and 6 wt. $\%$ ) for UV/IR, visible light and SHGC.

\section{ACKNOWLEDGMENTS}

This study was supported by the National Natural Science Foundation of China (Grant Nos.: $61565010 ; 11762009 ; 61865007)$.

\section{REFERENCES}

1. K. Lundgren and T. Kjellstrom, Sustainability 5, 3116 (2013).
2. A. Lopez, L. Sanchez, F. Doctor, H. Hagras, and V. Callaghan, IEEE Int. Conf. 1-7, 42 (2004).

3. G. Ramírez-Díaz, V. Nadal-Mora, and J. Piechocki, Renew. Sust. Energy Rev. 51, 138 (2015).

4. Y.Z. Lu, B. Zhu, J. Wang, Y.M. Zhang, and J.J. Li, Int. J. Energy Res. 40, 717 (2016).

5. M.M. Kabir and D.E. Demirocak, Int. J. Energy Res. 41, 1963 (2017).

6. A.M. Omer, Renew. Sust. Energy Rev. 12, 2265 (2008).

7. H.W. He, H. Jia, W.W. Huo, and M. Yan, Energy Procedia 105, 2518 (2017).

8. Y. Song, S. Wu, and Y.Y. Yan, Int. J. Low. Carbon Technol. 10,305 (2015).

9. B.P. Jelle, A. Gustavsen, and R. Baetens, J. Build. Phys. 34, 99 (2010).

10. C.S. Long, H.H. Lub, D.F. Lii, and J.L. Huang, Surf. Coat. Technol. 284, 75 (2015).

11. F. Shi, J.X. Liu, X.L. Dong, Q. Xu, J.Y. Lou, and H.C. Ma, J. Mater. Sci. Technol. 30, 342 (2014).

12. L.H. Xiao, Y.C. Su, X.Z. Zhou, H.Y. Chen, J. Tan, T. Hu, J. Yan, and P. Peng, Appl. Phys. Lett. 101, 041913 (2012).

13. Y. Wua, L. Zhang, G.H. Min, H.S. Min, B.H. Gao, H.H. Liu, S.L. Xing, and T. Pang, Appl. Surf. Sci. 384, 413 (2016).

14. X.M. Luo, P. Zhang, R. Liu, W.H. Li, B.H. Ge, and M. Cao, Polym. Int. 65, 415 (2016).

15. S. Dubin, S. Gilje, K. Wang, V.C. Tung, K. Cha, A.S. Hall, J. Farrar, R. Varshneya, Y. Yang, and R.B. Kaner, ACS Nano 4, 3845 (2010).

16. G.J. Zhang, Z.H. Chen, X.R. Zeng, F. Yu, and J. Wang, J. Coat. Technol. Res. 8, 505 (2011).

17. H. Huang, M.H. Ng, Y.L. Wu, and L.B. Kong, Mater. Des. 88, 384 (2015).

18. S. Ray, U. Dutta, R. Das, and P. Chatterjee, J. Phys. D Appl. Phys. 40, 2445 (2007).

19. H.F. Zhou, H. Wang, X.Y. Tian, K. Zheng, Z.F. Wu, X. Ding, and X.Z. Ye, Compos. Sci. Technol. 94, 105 (2014). 
20. T. Hu, Y.C. Su, S.D. Liu, H.B. Tang, S.J. Mu, and Z.X. Hu, Appl. Phys. A-Mater. 116, 1951 (2014).

21. T. Hu, Y.C. Su, I.R. Baxendale, J. Tan, H.B. Tang, L.H. Xiao, F. Zheng, and P. Ning, Curr. Appl. Phys. 17, 584 (2017).

22. H. Gliemann, A.T. Almeida, D.F.S. Petri, and T. Schimmel, Surf. Interface Anal. 39, 1 (2007).

23. B.H. Liu, J. Xu, and Y.B. Li, Adv. Mater. Sci. Eng. 16, 1 (2014).

24. S. Foghmoes, F. Teocoli, K. Brodersen, T. Klemenso, and M.D. Negra, J. Eur. Ceram. Soc. 36, 3441 (2016). https://doi. org/10.1016/j.jeurceramsoc.2016.05.043.
25. Y.V. Kuznetsova and A.A. Rempel, Inorg. Mater. 51, 215 (2015).

26. O. Prakash and A. Moitra, Comput. Mater. Sci. 295-297, 2615 (2011)

27. T.T. Liu, H. Luo, and J. Ma, Eur. Phys. J. E 39, 24 (2016).

28. P.K. Giri, S. Bhattacharyya, D.K. Singh, R. Kesavamoorthy, B.K. Panigrahi, and K.G.M. Nair, J. Appl. Phys. 102, 093515 (2007).

Publisher's Note Springer Nature remains neutral with regard to jurisdictional claims in published maps and institutional affiliations. 